\title{
EDITORIAL
}

\section{Is IgG galactosylation the relevant factor for pregnancy-induced remission of rheumatoid arthritis?}

\author{
Frauke Förger* and Monika Østensen \\ See related research by van de Geijn et al., http://arthritis-research.com/content/11/6/R193
}

\begin{abstract}
During pregnancy, most patients with rheumatoid arthritis (RA) experience spontaneous improvement of their disease activity. Among the soluble candidates that have been investigated in search for the most relevant disease-remitting factor are the galactosylation levels of immunoglobulin $\mathrm{G}$ (lgG). In RA, a higher percentage of IgG lacking the terminal galactose residues, thought to play a pro-inflammatory role, is found. During pregnancy, however, IgG galactosylation levels increase and correlate with improved disease activity. The question remains whether the increase in IgG galactosylation during pregnancy is a mere epiphenomenon or a true remission-inducing factor.
\end{abstract}

In a recent issue of Arthritis Research \& Therapy, van de Geijn and colleagues [1] analyse the role of immunoglobulin G (IgG) galactosylation as a disease-remitting factor in pregnant patients with rheumatoid arthritis (RA). The pregnancy-induced improvement of RA has intrigued researchers since the time of Philipp Hench, who in searching for the remission-inducing factor discovered cortisone [2]. Unfortunately, neither the corticosteroids nor other circulating factors, such as sex steroid hormones or $\alpha-2$ pregnancy-associated globulin, proved to be the crucial players that could explain remission of RA in pregnant patients [3-5]. Therefore, the search for a factor or a combination of factors able to substantially mitigate symptoms of RA continued.

Changes in the percentage of IgG molecules lacking the terminal galactose units in the oligosaccharide chains

*Correspondence: Frauke.Foerger@insel.ch

Department of Rheumatology, Clinical Immunology and Allergology; University Hospital and University of Bern, $\mathrm{CH}-3010$ Bern, Switzerland attached to $\mathrm{CH} 2$ regions have been investigated as a possible explanation for amelioration of RA during pregnancy. The percentage of agalactosyl IgG (Gal-0) varies as a function of age in normal healthy individuals; however, in patients with RA, Gal-0 levels exceed agerelated normal values [6]. Two studies including 8 and 23 RA patients during pregnancy and postpartum found a correlation between changes in disease activity and changes in Gal-0 $[7,8]$. However, no significant difference was seen in the galactosylation levels between the 11 patients that experienced remission and the 12 patients with persistent active disease during pregnancy [8].

In their study, van de Geijn and colleagues [1] report on immunoglobulin galactosylation in 148 RA patients and 32 healthy controls during and after pregnancy. Applying the European League Against Rheumatism response criteria, the authors were able to identify a pregnancy responder group as well as a non-responder group of RA patients. As shown previously [7], IgG galactosylation increased during pregnancy and decreased postpartum in healthy controls as well as in RA patients, showing this to be a pregnancy related phenomenon. High galactosylation levels were associated with low disease activity of RA and vice versa. Interestingly, increased galactosylation levels during pregnancy were more pronounced in the RA responder group and resembled those of healthy women.

Does this study provide a final answer for the spontaneous improvement or remission occurring in most pregnant RA patients? Changes in disease activity and changes in circulating proteins during and after pregnancy could be merely coincidental. The major problem of previous investigations has been the very limited number of pregnant RA patients studied and the inability to compare groups of remitting and nonremitting RA patients. A true candidate should either have a direct or inverse correlation with RA disease activity. Furthermore, levels should be significantly different in patients that improve and those that do not. In the study of van de Geijn and colleagues [1], the levels 
of IgG galactosylation in RA patients that spontaneously improved and those that did not were significantly different. Thus far, the study has confirmed that levels of disease activity and levels of IgG galactosylation are inversely correlated, and this is independent of pregnancy. Changes in disease activity and levels of IgG galactosylation occurred simultaneously, which leaves the question open whether the relationship is causal or an epiphenomenon.

Obviously, IgG galactosylation is a pregnancy-related phenomenon most abundant in healthy pregnant women and possibly necessary to protect the fetus against antipaternal antibodies. Whether it can modify autoimmune disease remains an open question. Elevated agalactosyl IgG levels are also present in Crohn's disease patients [9]. However, these patients experience either no or only mild improvement of their disease during pregnancy [10]. Thus, the pregnancy-related increase of IgG galactosylation does not seem to play a relevant role in the activity of Crohn's disease.

The role of IL-6 in the modification of IgG galactosylation is of interest. Low concentrations of IL- 6 were shown to increase IgG galactosylation via modulation of glycosyltransferase activity [11]. However, high levels of IL-6 decreased the glycosyltransferase activity. Therefore, IL-6 of placental origin could induce increased IgG galactosylation levels during pregnancy whereas elevated IL-6 levels of active RA patients could account for lower IgG galactosylation rates.

The final proof for a causal relationship between pregnancy-induced improvement of RA and IgG galactosylation levels is still lacking. Other factors contributing to maternal/fetal tolerance, such as Th2 cytokines, HLA-G/HLA-E, fetal antigens, inhibitory costimulatory molecules, complement regulators, regulatory $\mathrm{T}$ cells, and indoleamine 2,3-dioxygenase-producing dendritic cells, could be involved, acting further upstream.

Abbreviations

$\lg \mathrm{G}=$ immunoglobulin $\mathrm{G} ; \mathrm{lL}=$ interleukin; RA = rheumatoid arthritis.
Competing interests

The authors declare that they have no competing interests.

Published: 24 February 2010

\section{References}

1. van de Geijn FE, Wuhrer M, Selman MHJ, Willemsen SP, de Man YA, Deelder AM, Hazes JMW, Dolhain RJEM: Immunoglobulin G galactosylation and sialylation are associated with pregnancy-induced improvement of rheumatoid arthritis and the postpartum flare: results from a large prospective cohort study. Arthritis Res Ther 2009, 11:R193.

2. Hench PS, Kendall EC, Slocumb CH, Polley HF: The effect of a hormone of the adrenal cortex (17-hydroxy-11-dehydrocorticosterone: Compound E) and of pituitary adrenocorticotrophic hormone on rheumatoid arthritis. Preliminary report. Proc StaffMeet Mayo Clin 1949, 24:181-197.

3. Van den Brink HR, van Everdingen AA, van Wijk MJ, jcobs JW, Bijlsma JW: Adjuvant oestrogen therapy does not improve disease activity in postmenopausal patients with rheumatoid arthritis. Ann Rheum Dis 1993, 52:862-865.

4. Unger A, Kay A, Griffin AJ, Panayi G: Disease activity and pregnancy associated alpha2-glycoprotein in rheumatoid arthritis during pregnancy. BMJ 1983, 286:750-752.

5. Østensen M, Husby G: Pregnancy-associated alpha-glycoprotein, oral contraceptives, and rheumatoid arthritis. Lancet 1983, 18:1391.

6. Rahman A, Isenberg D: Does it take sugar? A clinical role for measuring the glycosylation of lgG? Ann Rheum Dis 1995, 54:689-691.

7. Rook GA, Steele J, Brealy R, Whyte A, Isenberg D, Sumar N, Nelson JL, Bodman $K B$, Young $A$, Roitt IM, et al.: Changes in IgG glycoform levels are associated with remission of arthritis during pregnancy. J Autoimmun 1991, 4:779-794.

8. Alavi A, Arden N, Spector TD, Axford JS: Immunoglobulin G glycosylation and clinical outcome in rheumatoid arthritis during pregnancy. J Rheum 2000, 27:1379-1385.

9. Dube R, Rook GAW, Steele J, Brealey R, Dwek R, Rademacher T, Lennard-Jones $\mathrm{J}$ : Agalactosyl lgG in inflammatory bowel disease: correlation with C-reactive protein. Gut 1990, 31:431-434.

10. Agret F, Cosnes J, Hassani Z, Gornet JM, Gendre JP, Lemann M, Beaugerie L: Impact of pregnancy on the clinical activity of Crohn's disease. Aliment Pharmacol Ther 2005, 21:509-513.

11. Miranda S, Canellada A, Gentile T, Margni R: Interleukin-6 and dexamethasone modulate in vitro asymmetric antibody synthesis and UDP-GIc glycoprotein glycosyltransferase activity. J Reprod Immuno/ 2005, 66:141-150.

doi:10.1186/ar2919

Cite this article as: Förger F, Østensen M: Is IgG galactosylation the relevant factor for pregnancy-induced remission of rheumatoid arthritis? Arthritis

Research \& Therapy 2010, 12:108. 